\title{
Center-of-mass response of confined systems
}

\author{
L. F. Lemmens \\ Departement Natuurkunde, Universiteit Antwerpen RUCA, Groenenborgerlaan 171, B2020 Antwerpen, Belgium \\ F. Brosens and J. T. Devreese \\ Departement Natuurkunde, Universiteit Antwerpen UIA, Universiteitsplein 1, B2610 Antwerpen, Belgium
}

(Received 11 September 1998)

\begin{abstract}
For confined systems of identical particles, either bosons or fermions, we argue that the parabolic nature of the confinement potential is a prerequisite for the nondissipative character of the center-of-mass response to a uniform probe. For an excitation in a parabolic confining potential, the half-width of the density response function depends nevertheless quantitatively on properties of the internal degrees of freedom, as is illustrated here for an ideal confined gas of identical particles with harmonic interparticle interactions.
\end{abstract}

[S1050-2947(99)05004-0]

PACS number(s): 03.75.Fi, 05.30.Jp, 32.80.Pj

\section{INTRODUCTION}

In the present paper we study the response of a confined system of spin-polarized identical particles if a uniform but time-dependent force is applied. Our method is based on the Feynman-Kac functional representing the propagator of distinguishable particles, followed by the projection of this functional on the irreducible representations of the permutation group as required for identical particles. The projection introduced in [1] is applied on confined systems. Details have been given earlier [2] to derive the thermodynamical properties and the static response functions for bosons and for fermions. An application of the method to real systems can be found in Ref. [3] for rubidium.

The response function is calculated for a spatially homogeneous but time-dependent force. It is shown that if the confinement potential is a polynomial of order 2 , the motion of the center of mass does not induce transitions between the modes representing the relative motion of the internal degrees of freedom. This follows from the independence of these degrees of freedom, a property known [5] for harmonic systems. The analog in extended systems is the Kohn theorem [4], well known for the cyclotron resonance in an interacting electron gas. The connection to the response of centerof-mass excitation becomes especially relevant if the twobody interaction only depends on the distance vector between the positions. If one of these conditions is not satisfied, we shall show that the homogeneous external force couples to the internal degrees of freedom. Even if both conditions are satisfied the shape of the response function still depends on the characteristics of the internal degrees of freedom, typically the frequency of the internal oscillation modes and the number of particles in the well. This suggests that these characteristics can be estimated from the shape of the response to a homogeneous time-dependent force. For instance, monitoring the density of a confined system while changing the direction of the gravitational force on the center of mass or changing slightly the form of the confinement potential gives information on the internal degrees of freedom and the number of particles confined in the system.

In view of the experimental progress made [6-9] on the Bose-Einstein condensed systems, the present response prop- erty of a perfect harmonic confined system could be relevant to check the parabolicity of the trap. Further recent experiments [10] use strong deviations from the parabolic confinement potential to probe the time-dependent behavior of the confined atoms in a condensed phase. In this respect it seems important to know the implications of perfect parabolicity. Of course, the response of the system to such a local perturbation of the density would be of major importance.

The paper is organized as follows. In Sec. II the formal response theory is developed. In Sec. III we illustrate the response properties for the exactly soluble model of a confined gas with harmonic interparticle interactions. In the last section a discussion and the conclusions are given.

\section{PARABOLICITY AND THE KOHN THEOREM}

In a $3 N$-dimensional configuration space with $\mathbf{r}_{j}(j$ $=1,2, \ldots, N)$ denoting the positions of the $N$ particles, the position of the center of mass is defined as $\mathbf{R}$ $=(1 / N) \sum_{j=1}^{N} \mathbf{r}_{j}$. For a quadratic confinement potential the center of mass can be introduced as follows:

$$
\sum_{j=1}^{N} \mathbf{r}_{j}^{2}=N \mathbf{R}^{2}+\sum_{j=1}^{N}\left(\mathbf{r}_{j}-\mathbf{R}\right)^{2}
$$

From mathematical statistics it is well known that for Gaussian distributions the average $\mathbf{R}$ can take any value without affecting the value of $\sum_{j=1}^{N}\left(\mathbf{r}_{j}-\mathbf{R}\right)^{2}$, i.e., the center of mass could be independent of the deviations from that center. This remains true in a physical system when the twobody potential depends only on the distance between particles, because $\sum_{j, l=1}^{N} V\left(\mathbf{r}_{j}-\mathbf{r}_{l}\right)=\sum_{j, l=1}^{N} V\left(\left(\mathbf{r}_{j}-\mathbf{R}\right)-\left(\mathbf{r}_{l}\right.\right.$ $-\mathbf{R})$ ). In the harmonic model the following two-body potential is used:

$$
\frac{\gamma}{4} \sum_{j, l=1}^{N}\left(\mathbf{r}_{j}-\mathbf{r}_{l}\right)^{2}=\frac{\gamma N}{2} \sum_{j=1}^{N}\left(\mathbf{r}_{j}-\mathbf{R}\right)^{2} .
$$

This two-body potential allows for exact solutions of the propagator and the projection on the symmetric or antisymmetric irreducible representation of the permutation group can be carried out. 
Introducing a homogeneous time-dependent force

$$
\mathbf{f}(\tau) \cdot \sum_{j=1}^{N} \mathbf{r}_{j}=N \mathbf{f}(\tau) \cdot \mathbf{R}
$$

the potential confining $N$ particles and the interaction of the particles with each other and the external field are specifying the system for which the response will be calculated. Atomic units with $\hbar=m=1$ are used throughout this paper.

If the particles were distinguishable, the Euclidean-time propagator $K_{D}\left(\overline{\mathbf{r}}^{\prime \prime}, \tau \mid \overline{\mathbf{r}}^{\prime}, 0\right)$ for this interacting system can be obtained from a straightforward generalization of our calculation in Ref. [2]. Denoting by $\overline{\mathbf{r}} \in \mathbb{R}^{3 N}$ the set of position vectors $\mathbf{r}_{1}, \ldots, \mathbf{r}_{N}$, the result turns out to be

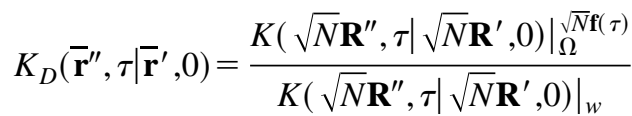

$$
\begin{aligned}
& \times\left.\prod_{j=1}^{N} K\left(\mathbf{r}_{j}^{\prime \prime}, \tau \mid \mathbf{r}_{j}^{\prime}, 0\right)\right|_{w},
\end{aligned}
$$

where $\left.K\left(\mathbf{r}_{\tau}, \tau \mid \mathbf{r}_{0}, 0\right)\right|_{w}$ denotes the well-known propagator of a three-dimensional harmonic oscillator of frequency $w$ :

$$
\begin{aligned}
\left.K\left(\mathbf{r}_{\tau}, \tau \mid \mathbf{r}_{0}, 0\right)\right|_{w}= & \sqrt{\frac{w}{2 \pi \sinh w \tau}}^{3} \\
& \times \exp \left[-\frac{w}{2} \frac{\left(\mathbf{r}_{\tau}^{2}+\mathbf{r}_{0}^{2}\right) \cosh w \tau-2 \mathbf{r}_{\tau} \cdot \mathbf{r}_{0}}{\sinh w \tau}\right]
\end{aligned}
$$

The quantity $K\left(\mathbf{R}^{\prime \prime}, \tau \mid \mathbf{R}^{\prime}, 0\right){ }_{\Omega}^{[\mathbf{f}(\tau)]}$ denotes the propagator of a three-dimensional harmonic oscillator of frequency $\Omega$ in the presence of a driving force $\mathbf{f}(\tau)$. If the driving force acts in the real time $t$, the complex time $\tau=\beta+i t$ has to be introduced, with $\beta=1 / k_{B} T$ with $k_{B}$ the Boltzmann constant and $T$ the temperature. This propagator can be obtained in a direct way using the functional integration techniques illustrated in [2], leading to the following result:

$$
\begin{aligned}
\left.K\left(\mathbf{R}^{\prime \prime}, \tau \mid \mathbf{R}^{\prime}, 0\right)\right|_{\Omega} ^{\mathbf{f}(\tau)=} & \left.K\left(\mathbf{R}^{\prime \prime}, \tau \mid \mathbf{R}^{\prime}, 0\right)\right|_{\Omega} \exp \left(-\frac{2}{\Omega} \frac{\sinh \left(\frac{1}{2} \Omega \beta\right)}{\cosh \left(\frac{1}{2} \Omega \beta\right)} \int_{0}^{t} \int_{0}^{s} \mathbf{f}(s) \cdot \mathbf{f}(\sigma) \sin [\Omega(t-s)] \sin [\Omega(s-\sigma)] d s d \sigma\right) \\
& \times \exp \left(i \mathbf{R}^{\prime} \cdot \int_{0}^{t} \mathbf{f}(s) \frac{\cos \left[\Omega\left(s-t+\frac{1}{2} i \beta\right)\right]}{\cosh \left(\frac{1}{2} \Omega \beta\right)} d s-i \mathbf{R}^{\prime \prime} \cdot \int_{0}^{t} \mathbf{f}(s) \frac{\cos \left[\Omega\left(s-t-\frac{1}{2} i \beta\right)\right]}{\cosh \left(\frac{1}{2} \Omega \beta\right)} d s\right) .
\end{aligned}
$$

From the structure of the propagator $K_{D}\left(\overline{\mathbf{r}}^{\prime \prime}, \tau \mid \overline{\mathbf{r}}^{\prime}, 0\right)$ for distinguishable particles, it is clear that projection on the representations of the permutation group will not affect the quotient of propagators that contains the center of mass. Therefore, the propagator $K_{I}\left(\overline{\mathbf{r}}^{\prime \prime}, \tau \mid \overline{\mathbf{r}}^{\prime}, 0\right)$ for identical particles is given by

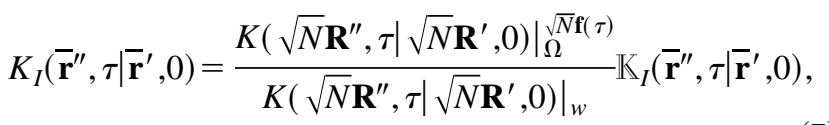

where $\mathbb{K}_{I}\left(\overline{\mathbf{r}}^{\prime \prime}, \tau \mid \overline{\mathbf{r}}^{\prime}, 0\right)$ accounts for the permutations of the particles, and is discussed extensively in [2]. Although the present derivation has been given for the fully harmonic model (harmonic confinement and harmonic interaction), the line of the derivation remains valid for more general twobody interactions. Indeed, the separation between the propagation of the center of mass and of the other degrees of freedom is unaffected by the introduction of the two-body potential if it only depends on the difference vectors $\mathbf{r}_{j}$ $-\mathbf{r}_{l}$.

\section{RESPONSE TO A UNIFORM FORCE}

Knowing the propagator of the interacting many-particle system, the density response can be calculated in a straightforward way as the following expectation value:

$$
\begin{gathered}
n^{\mathbf{f}}(\mathbf{r}, t)=\frac{1}{N} \sum_{j=1}^{N}\left\langle\delta\left(\mathbf{r}-\mathbf{r}_{j}\right)\right\rangle=\left(\frac{1}{2 \pi}\right)^{3} \int n_{\mathbf{q}}^{\mathbf{f}}(t) \exp (-i \mathbf{q} \cdot \mathbf{r}) d \mathbf{q} \\
n_{\mathbf{q}}^{\mathbf{f}}(t)=\frac{1}{N} \sum_{j=1}^{N}\left\langle\exp \left(i \mathbf{q} \cdot \mathbf{r}_{j}\right)\right\rangle .
\end{gathered}
$$

The averages $\langle A(\overline{\mathbf{r}}, t)\rangle$ are defined as

$$
\langle A(\overline{\mathbf{r}}, t)\rangle=\frac{1}{Z(\beta)} \int d \overline{\mathbf{r}} A(\overline{\mathbf{r}}) K_{I}(\overline{\mathbf{r}}, \beta+i t \mid \overline{\mathbf{r}}, 0),
$$

where $Z(\beta)$ is the partition function for the system without external force at inverse temperature $\beta$. Performing this average means the repetitive calculation of Gaussian integrals leading to the following result: 


$$
\begin{aligned}
& n_{\mathbf{q}}^{\mathbf{f}}(t)=n_{\mathbf{q}}^{\mathbf{f}}(0) \exp \left(-i \mathbf{q} \cdot \int_{0}^{t} \mathbf{f}(s) \frac{\sin \Omega(t-s)}{\Omega} d s\right), \\
& n_{\mathbf{q}}^{\mathbf{f}}(0)=\exp \left\{-\frac{\mathbf{q}^{2}}{4 N}\left[\frac{N-1}{w} \frac{\cosh \frac{1}{2} w \beta}{\sinh \frac{1}{2} w \beta}\right.\right. \\
& \left.\left.+\frac{1}{\Omega} \frac{\cosh \frac{1}{2} \Omega \beta}{\sinh \frac{1}{2} \Omega \beta}\right]\right\} \text {. }
\end{aligned}
$$

The response function $n^{\mathbf{f}}(\mathbf{r}, t)$ is then obtained by a Fourier transform leading to a Gaussian density distribution function

$$
\begin{aligned}
n^{\mathbf{f}}(\mathbf{r}, t)= & \frac{1}{{\sqrt{2 \pi S^{2}}}^{3}} \\
& \times \exp \left\{-\frac{\left.\left\{\mathbf{r}+(1 / \Omega) \int_{0}^{t} \mathbf{f}(s) \sin [\Omega(t-s)] d s\right\}^{2}\right\}}{2 S^{2}}\right\},
\end{aligned}
$$

in which the variance not only depends on the frequency $\Omega$ of the confining potential, but also on the frequency $w$ of the internal degrees of freedom:

$$
S^{2}=\frac{1}{2}\left(\frac{N-1}{w N} \operatorname{coth} \frac{w \beta}{2}+\frac{1}{\Omega N} \operatorname{coth} \frac{\Omega \beta}{2}\right) .
$$

In the absence of interparticle interactions, one readily obtains $\left.S^{2}\right|_{w=\Omega}=\left(\operatorname{coth} \frac{1}{2} \Omega \beta\right) /(2 \Omega)$, which in the lowtemperature limit $\beta \rightarrow \infty$ gives the variance of a harmonic oscillator with frequency $\Omega$.

In the average density response

$$
\langle\mathbf{r}(t)\rangle=\int \mathbf{r} n^{\mathbf{f}}(\mathbf{r}, t) d \mathbf{r}=-\frac{1}{\Omega} \int_{0}^{t} \mathbf{f}(s) \sin [\Omega(t-s)] d s
$$

one clearly sees the resonant structure in the presence of an oscillating driving force $\mathbf{f}(s)=\mathbf{f}_{v} \sin v s$ with a specific frequency $v$ :

$$
\left.\langle\mathbf{r}(t)\rangle\right|_{\mathbf{f}(s)=\mathbf{f}_{v} \sin v s}=-\frac{\mathbf{f}_{v}}{\Omega} \frac{\Omega \sin t v-v \sin \Omega t}{\Omega^{2}-v^{2}} .
$$

This expectation value of the position does not depend on the parameters of the internal degrees of freedom, as required by the Kohn theorem.

\section{DISCUSSION}

From the derivation of the density response to a uniform time-dependent force, it is clear that the factorization (4) is crucial to obtain a linear relation between the density and the applied force that does not depend on the internal degrees of freedom. Higher-order terms in the confinement potential will spoil this relation. A term of the third degree in the confinement potential already introduces a contribution $V_{\text {conf }} \sim 3 \mathbf{R} \Sigma_{j=1}^{N}\left(\mathbf{r}_{j}\right)^{2}$, that mixes the center-of-mass motion with that of the internal degrees of freedom, and makes transitions possible in the internal degrees of freedom leading eventually to dissipative behavior of the center-of-mass excitation.

Furthermore, the Gaussian nature of the density response may allow one to check the parabolic character of the well by measuring the mean density and comparing it with the trap frequencies. The standard deviations of these measurements contain information about the excitation frequencies of the internal degrees of freedom and the number of particles contained in the well. The response of a center-of-mass mode behaving according to the Kohn theorem contains information about the internal degrees of freedom. Its resonance structure reveals the parabolic quality of the well.

It should be noted that the statistics of the internal degrees of freedom does not enter in the expressions. Therefore the response of a parabolic well to a uniform external force does not distinguish between fermions or bosons merely because it only applies to the center of mass.

\section{ACKNOWLEDGMENTS}

This work was performed in the framework of FWO Project Nos. 1.5.729.94, 1.5.545.98, G. 0287.95, G.0071.98, and WO.073.94N (Wetenschappelijke Onderzoeksgemeenschap over "'Laagdimensionele Systemen'), the "Interuniversitaire Attractiepolen-Belgische Staat, Diensten van de Eerste Minister-Wetenschappelijke, Technische en Culturele Aangelegenheden,' and in the framework of the BOF NOI projects of the Universiteit Antwerpen. One of the authors (F.B.) acknowledges the FWO (Fonds voor Wetenschappelijk Onderzoek-Vlaanderen) for financial support.
[1] R. P. Feynman, Statistical Mechanics, A Set of Lectures (W. A. Benjamin, Reading, MA, 1972).

[2] F. Brosens, J. T. Devreese, and L. F. Lemmens, Phys. Rev. E 55, 227 (1997); 55, 6795 (1997); 57, 3871 (1998); 58, 1634 (1998).

[3] J. Tempere, F. Brosens, L. F. Lemmens, and J. T. Devreese,
Solid State Commun. 107, 51 (1998).

[4] W. Kohn, Phys. Rev. 123, 1242 (1962).

[5] A. Skyrme and R. J. Elliott, Proc. R. Soc. London, Ser. A 232, 566 (1955).

[6] M. H. Anderson, J. R. Ensher, M. R. Matthews, C. E. Wieman, and E. A. Cornell, Science 269, 198 (1995). 
[7] K. B. Davis, M.-O. Mewes, M. R. Andrews, N. J. Van Druten, D. S. Durfee, D. M. Kurn, and W. Ketterle, Phys. Rev. Lett. 75, 3969 (1995).

[8] C. C. Bradley, C. A. Sackett, J. J. Tollett, and R. G. Hulet, Phys. Rev. Lett. 75, 1687 (1995).
[9] C. C. Bradley, C. A. Sackett, and R. G. Hulet, Phys. Rev. Lett. 78, 985 (1997).

[10] D. M. Stamper-Kurn, M. R. Andrews, A. P. Chikkatur, S. Inouye, H.-J. Miesner, J. Stenger, and W. Ketterle, Phys. Rev. Lett. 80, 2027 (1998). 